\title{
Neuroendocrine Tumors of the Gastrointestinal Tract
}

\author{
Detlef K. Bartsch ${ }^{a}$ Hans Scherübl ${ }^{b}$ \\ ${ }^{a}$ Department of Visceral, Thoracic and Vascular Surgery, University Hospital Marburg, Marburg, Germany; \\ ${ }^{b}$ Departments of Gastroenterology, Gastrointestinal Oncology and Infectious Diseases, Vivantes Klinikum Am Urban, Berlin, Germany
}

Neuroendocrine neoplasms (NEN) are heterogeneous tumors with a common phenotype. Two groups of NEN are distinguished: well-differentiated, low-proliferating NEN, called neuroendocrine tumors (NET) or carcinoids, and poorly differentiated, highly proliferating (G3) NEN, called small or large cell neuroendocrine carcinomas (NEC G3). NET exhibit a wide range of clinical behaviors due to the secretion of hormones, most often serotonin, which creates non-specific but debilitating systemic symptoms such as diarrhea, bronchospasm, flushing, and cardiac valve disease. Concerning therapeutic options, there is quite some overlap between pancreatic NET (pNET) G2 and pancreatic NEC G3. Paying attention to this clinical observation, the World Health Organization (WHO) has now introduced the new NET G3 category in its recent classification of pancreatic NEN.

In terms of epidemiology, an impressive rise of clinically detected, mostly early-stage asymptomatic NET could be demonstrated in the last 40 years. NET are now more prevalent than stomach and exocrine pancreatic cancers combined. Better awareness and improved diagnostic techniques have contributed to the clinically observed rise in incidence and prevalence.

NET of the stomach, duodenum (except gastrinoma), pancreas, appendix, or rectum that are $\leq 1 \mathrm{~cm}$ in size and well-differentiated with WHO grade 1 (G1, Ki-67 $\leq 2 \%$ ) can be considered as 'early' NET; they have an excellent prognosis. Endoscopic resection is the treatment of choice in asymptomatic NET G1 of the stomach, duodenum, and rectum that are $\leq 10 \mathrm{~mm}$ in size, do not infiltrate the muscularis propria (T1), and show no angioinvasion (V0, L0). Histologically proven, early pNET G1 $(<20 \mathrm{~mm})$ can be managed conservatively, too.

Curative surgery is the treatment of choice for localized NEN. Individualized surgical procedures performed by experienced NET surgeons are based on tumor biology and possible multifocality. In intestinal NET with lymph node metastases along the mesenteric vessels, as much tumor volume as possible should be resected, thus avoiding major intraoperative complications and postoperative short bowel syndrome. Pancreatic NEN, especially small tumors $<4$ $\mathrm{cm}$ in the left pancreas, can be approached with the minimally invasive technique nowadays. Neuroendocrine liver metastases are challenging to the surgeon, the interventional radiologist, the nuclear medicine physician, and the oncologist, and are often treated by a multimodal interdisciplinary approach.

Somatostatin receptor (SSTR) positron emission tomography/ computed tomography (PET/CT) using ${ }^{68} \mathrm{Ga}$-labeled somatostatin analogs is the mainstay for the evaluation of SSTR status in NET. This translates into better SSTR-guided treatment strategies with increasing evidence of peptide receptor radionuclide therapy (PRRT) as a very effective and safe treatment option for advanced or progressive SSTR-positive NET. There are significant benefits in progression-free and overall survival as well as an improvement in clinical condition to be found. In patients with progressive SSTRpositive NET, fractionated, personalized PRRT results in good therapeutic responses with no significant severe hematological and/or renal toxicity, therefore improving the quality of life of the patients.

Advanced metastasized NET of the small intestine usually present with low proliferation and are treated in the first line with somatostatin analogs. If progression occurs, PRRT is the treatment of choice in SSTR-positive NET. For SSTR-negative NET one can add interferon alpha or change the medical treatment to everolimus. Rectal NET are often small when they metastasize. If SSTR-positive, they can be treated with PRRT, and another option is of course everolimus. Colonic NET are more aggressive than the rest of intestinal NET and will be treated with everolimus, sometimes in combination with somatostatin analogs based on positive scintigraphy or PET. Another option is a cytotoxic agent such as streptozotocin plus 5-fluorouracil (5-FU) or temozolomide plus capecitabine. The most aggressive tumors, i.e. NEC G3, are treated with platin-based therapy plus etoposide if they present with a proliferation rate $>55 \%$.

Metastasized duodenal NET and pNET are treated either with cytotoxic agents, streptozotocin plus 5-FU, or temozolomide plus capecitabine or with targeted agents such as everolimus. Somatostatin analogs are established in well-differentiated pNET with a Ki-67 $<10 \%$. For pNET with a Ki-67 $<20 \%$, both the multikinase inhibitor sunitinib and the mTOR inhibitor everolimus are effective.

\section{KARGER}

(c) 2017 S. Karger GmbH, Freiburg

Fax +497614520714
Prof. Dr. med. Hans Scherübl

Klinik für Innere Medizin - Gastroenterologie, Gastrointestinale Onkologie und Infektiologie Vivantes-Klinikum Am Urban, Akademisches Lehrkrankenhaus der Charité

Dieffenbachstraße 1, 10967 Berlin, Germany

hans.scheruebl@ vivantes.de 
The past 15 years witnessed several new therapies that benefit patients with gastroenteropancreatic (GEP) NET. PRRT is a major breakthrough in the development of innovative systemic targeted therapies. Both earlier detection and new treatment options have significantly improved the survival and quality of life of NET patients in the last two decades. Ongoing clinical trials are now investigating radiolabeled SSTR antagonists as well as immune checkpoint inhibitors in patients with NEN.
The present issue of Visceral Medicine, comprising six articles of experts in the field [1-6] as well as an interdisciplinary discussion chaired by Frilling [7], aims to give the reader a current overview of the pathology of as well as treatment options for GEPNET, including endoscopy, surgery, medical therapy, and PRRT.

\section{References}

1 Klöppel G: Neuroendocrine neoplasms: dichotomy, origin and classifications. Visc Med 2017;33:DOI: $10.1159 / 000481390$.

2 Scherübl H, Cadiot G: Early gastroenteropancreatic neuroendocrine tumors: endoscopic therapy and surveillance. Visc Med 2017;33:DOI: 10.1159/000459404.

3 Selberherr A, Niederle MB, Niederle B: Surgical treatment of small intestinal neuroendocrine tumors G1/ G2. Visc Med 2017;33:DOI: 10.1159/000477786.
4 Fendrich V, Bartsch DK: Surgical therapy of sporadic pancreatic neuroendocrine neoplasias G1/G2. Visc Med 2017;33:DOI: 10.1159/000456630.

5 Öberg K: Medical therapy of gastrointestinal neuroendocrine tumors. Visc Med 2017;33:DOI: 10.1159/ 000475831.

6 Lee ST, Kulkarni HR, Singh A, Baum RP: Theranostics of neuroendocrine tumors. Visc Med 2017;33:DOI: $10.1159 / 000480383$.
7 Frilling A, Herrmann K, Kämmerer D, Toumpanakis C: Neuroendocrine tumors of the gastrointestinal tract (Interdisciplinary Discussion). Visc Med 2017;33:DOI: 10.1159/000475539. 